\title{
The Assessment of Leadership Outcomes in Capstone Projects Using Anonymous Peer Feedback
}

\author{
William D. Bishop \\ Department of Electrical and Computer Engineering \\ University of Waterloo \\ Waterloo, ON, N2L 3G1 \\ wdbishop@uwaterloo.ca
}

\begin{abstract}
Assessing the leadership abilities of engineering students for the purposes of accreditation and outcomes assessment is a particularly challenging task. For a few students, clear evidence of leadership will exist. These students naturally take on leadership roles within their teams. They have the complete confidence and support of their teammates. The team functions as a cohesive unit. In such a situation, an instructor can easily recognize the quality of leadership provided but may struggle with quantitatively assessing the leadership abilities exhibited.

In other cases, students arguably possess leadership abilities yet fail to demonstrate their abilities in a way that can be assessed by an instructor. Such students might demonstrate leadership within their team by taking on an undesirable task or by taking on a disproportionate share of the workload. If a student perceives that leadership is not required, a student might demonstrate great leadership by not taking on an obvious leadership role. Peer feedback can help an instructor identify such examples of leadership.

To enable proper assessment of leadership abilities, a suitable environment must be created such that all students have a natural opportunity to demonstrate their leadership skills in their own way. Such an environment is often created by a capstone project. Given the scope of a capstone project, all team members typically have several opportunities to take on a leadership role and/or demonstrate leadership. The challenge is to assess leadership quantitatively without forcing a change in the behaviour of the students. This paper examines the design and implementation of an anonymous peer feedback survey for the purpose of quantitatively assessing different measures of teamwork and leadership abilities in engineering students. This paper describes the
\end{abstract}

challenges that are associated with such an assessment process. This paper also discusses the advantages and disadvantages of the proposed assessment technique.

Keywords: Anonymous Peer Feedback, Leadership Assessment, Outcomes Assessment, Capstone Projects, Accreditation

\section{INTRODUCTION}

Engineering institutions across Canada are required to assess graduate attributes as part of the recently revised Canadian Engineering Accreditation Board (CEAB) accreditation process. Many of the desired attributes of our engineering graduates can be quantitatively assessed using an assignment, laboratory exercise, project or test. However, the assessment of some desired graduate attributes pose a greater challenge. In particular, the assessment of higher-order skills such as teamwork and leadership skills can be difficult to assess directly in the context of undergraduate engineering courses.

This paper presents an assessment technique used by the Department of Electrical and Computer Engineering at the University of Waterloo to assess the teamwork and leadership skills of students immediately prior to graduation. An anonymous online survey is conducted during the last two weeks of the capstone project course. Although the survey is voluntary, sufficient data is collected to provide a representative sample of graduate attributes for the purposes of outcome assessment and accreditation. By tracking changes in the results from year to year, the survey results can be used to guide continuous program improvement.

This paper begins by describing some of the many challenges of teamwork and leadership assessment in 
the context of an engineering undergraduate program. Next, it presents the design of a survey for the purpose of assessing teamwork and leadership skills. Observations based on the survey results for the Electrical and Computer Engineering Graduating Class of 2013 are presented. Finally, the paper concludes with a few ideas for future work.

\section{BACKGROUND}

As outlined in the 2011 Canadian Engineering Accreditation Board (CEAB) Accreditation Criteria and Procedures [1], institutions must demonstrate that graduates of engineering programs possess specified attributes under the following headings:

1) A knowledge base for engineering

2) Problem analysis

3) Investigation

4) Design

5) Use of engineering tools

6) Individual and team work

7) Communication skills

8) Professionalism

9) Impact of engineering on society and the environment

10) Ethics and equity

11) Economics and project management

12) Life-long learning

Some attributes represent discipline-specific skills. Examples of discipline-specific skills include a knowledge of engineering design principles and an understanding of the proper use of engineering tools. Other attributes represent higher-order skills. Examples of higher-order skills include the ability to demonstrate leadership and professionalism. This section provides additional background on the assessment of graduate attributes related to teamwork and leadership skills.

\subsection{Assessing Graduate Attributes}

The assessment of discipline-specific skills is generally a straightforward task in the context of an engineering undergraduate program. Graded course deliverables provide a direct means of assessing quantitatively the attributes of students prior to graduation. Deliverables can be designed to examine a particular graduate attribute of interest. Results can be compiled and analyzed to examine the performance of a program over time.

However, higher-order skills are often more challenging to assess as these skills are rarely the sole subject of a particular course or course deliverable. Direct assessment of a higher-order skill is not an easy task. Most often, higher order skills are assessed using indirect assessment techniques such as peer surveys or self-assessments.

\subsection{Individual and Team Work}

The CEAB specifies under the heading of individual and team work that graduates must possess "an ability to work effectively as a member and leader in teams, preferably in a multi-disciplinary setting" [1]. This attribute requires the demonstration of both teamwork and leadership abilities. The capstone project course provides an exceptional opportunity for assessing the teamwork and leadership abilities of students since students are required to work in teams to complete engineering design projects. Given the complexity of the projects, students naturally have many opportunities to demonstrate both their teamwork skills and their leadership skills.

\subsection{Teamwork Assessment}

Individual programs are allowed to determine the set of outcomes expected to adequately measure graduate attributes within the context of the CEAB accreditation framework. The Department of Electrical and Computer Engineering at the University of Waterloo expects its students to demonstrate the following components of teamwork skills during the capstone project course:

1) Participate in team activities

2) Help the team to focus

3) Have a positive attitude towards collaboration

4) Handle inter-personal conflict

5) Willingly accept suggestions from others in the team

For each of the components listed above, the achievement levels of students are rated using a system loosely similar to Bloom's Taxonomy [2]. The lowest level of student achievement is a level where students can demonstrate the desired attribute with help from a faculty member, teaching assistant, or fellow student. The highest level of student achievement is a level where students can demonstrate the ability to adapt their skills to a new environment such as a new team structure.

\subsection{Leadership Assessment}

Leadership assessment has recently been added to the capstone project course. It is quite natural to assess teamwork skills and leadership skills in the 
same course as the two skill sets are closely related. Leadership is best demonstrated within the context of a team environment.

One of the many challenges of leadership assessment is to define the components of leadership to be assessed. Effective team leadership can be demonstrated in many ways. For example, Dale Carnegie Training offers a Team Leadership App [3] that encourages team leaders to do the following:

1) Delegate tasks to insure they are completed

2) Run meetings that accomplish tasks

3) Enhance the confidence and enthusiasm of team members through recognition

4) Coach team members for positive results

For purposes of comparison, a recent Forbes Magazine article [4] describes effective leaders as possessing honesty, an ability to delegate, communication skills, a sense of humour, confidence, commitment, a positive attitude, creativity, intuition, and the ability to inspire. Numerous textbooks and websites examine the subject of effective leadership and the leadership skills necessary to be a great leader. Achieving a consensus on the skills required to be a leader is difficult.

At a minimum, the Department of Electrical and Computer Engineering at the University of Waterloo expects its students to demonstrate the following components of leadership skills during the capstone project course:

1) Initiate new tasks and activities

2) Accept responsibility for tasks

3) Inspire others to be productive

As is the case for teamwork assessment, the achievement levels of students are rated with respect to each of the components of the leadership outcome.

\section{ASSESSMENT CHALLENGES}

The assessment of teamwork and leadership skills is a challenging task for many reasons including the following:

1) Undergraduate courses do not always provide a suitable opportunity for students to demonstrate their teamwork and leadership skills

2) Teamwork and leadership skills are difficult for a course instructor to observe and assess directly

3) Course instructors might not have the best perspective to evaluate the teamwork and leadership skills of students

This section elaborates on each of these assessment challenges.

\subsection{Limited Opportunities for Assessment}

Teamwork and leadership skills are higher-order skills that are not discipline-specific. While it is certainly the case that engineering professions demand some mastery of teamwork and leadership, it is also true that such skills are not unique to engineering professions. Teamwork and leadership skills are a desirable outcome of all university programs. This is reinforced by the fact that the Undergraduate Degree Level Expectations (UDLEs) [5] formulated by the Ontario Council of Academic Vice-Presidents (OCAV) includes an expectation that all university students possess the ability to "exercise initiative, personal responsibility, and accountability in both personal and group contexts." This outcome is very similar to the one specified by the CEAB.

Despite the fact that teamwork and leadership skills are a desirable outcome of all university programs, specific courses on the subjects of teamwork and leadership are not a requirement of many university programs. Instead, these higher-order skills are taught in the context of undergraduate courses on disciplinespecific topics and the skills are evaluated indirectly. Such courses do not always provide a suitable opportunity for students to demonstrate their abilities.

\subsection{Limited Observability}

Unlike discipline-specific graduate attributes, performing a direct assessment of an individual's leadership abilities is practically impossible. In the context of an engineering undergraduate course, an instructor cannot easily devise an effective assignment, laboratory exercise, project, or test to directly evaluate leadership skills. Direct observation of leadership abilities may not even be possible for numerous reasons including the following:

1) Large class sizes may make direct observation of team behaviour difficult if not completely impractical,

2) Dominant leaders within a team may hinder the observation of leadership skills in less dominant team members, and

3) Leaders of exceptional teams may be perceived as strong leaders when compared to leaders of weak teams.

One can argue this is a problem of observability. While leadership may exist, it is difficult to observe directly. When it is observable, it is very difficult to observe without bias. One way to address the problem of observability is to assess leadership abilities indirectly through the use of a survey. Such an approach 
reduces bias through multiple peer assessments and ensures observability through direct contact with the individual. Arguably, teammates are in a good position to evaluate the leadership skills of a team member.

\subsection{Perspective}

Perspective is an important consideration when assessing teamwork and leadership skills. The perspective of a course instructor may differ substantially from the perspective of a fellow student. While both perspectives can provide useful insight into the leadership abilities of an individual, there is no denying that leadership is often best evaluated by those being led, provided that there is no incentive for providing an inaccurate evaluation. Similarly, teamwork, is best evaluated by other members of the same team.

\section{SURVEY DESIGN}

An online survey was created using the survey tool of the Desire2Learn learning management system at the University of Waterloo. The goal of this survey was to ask all 4th year students in the Department of Electrical and Computer Engineering about the quality of teamwork skills and leadership skills demonstrated by their fellow teammates during the completion of their capstone project. This section describes the design of the online survey.

\subsection{Survey Organization}

The survey was divided into two sections. The first section examined teamwork skills and the second section examined leadership skills. Each question was repeated four times to allow each student to comment on the performance of each team mate, assuming a maximum team size of 5 members.

\subsection{Ratings}

For each question in the survey, students were asked to assign one of five possible quality ratings ranging from high to low as follows:

1) Outstanding,

2) Very Good,

3) Good,

4) Satisfactory, and

5) Unsatisfactory.

This rating system was chosen to match the ratings used by the standardized rubrics for engineering design project assessment at the University of Waterloo [6].
To account for varying team sizes, students were also given a sixth option of assigning a rating of "Not Applicable". Students were instructed to indicate "Not Applicable" if their team had fewer members than the maximum supported by the survey.

\subsection{Teamwork Assessment}

For the purpose of teamwork assessment, students were asked to answer four questions with respect to each member of their team. The questions asked were the following:

1) Willingness to perform assigned tasks?

2) Willingness to assist with additional tasks?

3) Willingness to ask for help when needed?

4) Overall impression of teamwork skills?

The questions were ordered such that the assessment of the overall impression of teamwork skills was the last question posed. The purpose of this ordering was to provide students with some guidance on a few of the factors to consider when rating their overall impression of teamwork skills for a team member.

Additional assessment questions were considered but ultimately rejected. Given that the survey was voluntary, it was very important to keep the length of the survey short to ensure adequate participation. While the questions do not match exactly with the specific components chosen for the teamwork outcome, the questions do provide sufficient information on the teamwork skills of students to extrapolate the overall level of achievement.

\subsection{Leadership Assessment}

For the purpose of leadership assessment, students were asked to answer four questions with respect to each member of their team. The questions asked were the following:

1) Willingness to take initiatives?

2) Willingness to accept responsibility for tasks?

3) Willingness to inspire others to be productive?

4) Overall impression of leadership skills?

The order of the questions was set to provide students with some guidance on a few of the factors to consider when rating their overall impression of leadership skills for a team member. Many additional assessment questions could have been asked but given the desire to ensure adequate participation, only the questions indicated were selected for the survey. The leadership assessment questions closely match the leadership outcome components. 


\subsection{Compiling the Survey Results}

For the purpose of compiling the survey results, weights were assigned to each of the ratings as illustrated in Table 1 1 . This permitted the calculation of a single numeric grade for each assessment question.

\section{Table 1. Assessment Ratings and Their} Corresponding Weights

\begin{tabular}{||c|r||}
\hline \hline Rating & Weight \\
\hline \hline Outstanding & $100 \%$ \\
\hline Very Good & $75 \%$ \\
\hline Good & $50 \%$ \\
\hline Satisfactory & $25 \%$ \\
\hline Unsatisfactory & $0 \%$ \\
\hline \hline
\end{tabular}

For example, if a survey question had a total of 3 responses from students of "Outstanding" (100\%), "Very Good" (75\%), and "Satisfactory" (25\%), the overall assessment grade would simply be $66.7 \%$. This value is the arithmetic mean of the individual assessment grades.

\subsection{Survey Methodology}

Participation in the teamwork and leadership survey was voluntary. No grades were assigned for completing the survey. The survey was conducted anonymously to avoid any perception that the results might influence project grades. Students were given two weeks to complete the survey. It should be noted that the survey, out of necessity, was conducted near the end of the academic term. A low participation rate of approximately $20 \%$ was expected given all of these factors.

\section{OBSERVATIONS}

Due to the anonymous nature of the survey, the details of the student responses to each of the survey questions have not been included in this paper. However, some general observations can be made regarding the participation of students, quality of the responses, advantages of anonymous surveys, and disadvantages of anonymous surveys.

\subsection{Participation Rate}

The participation rate was relatively low but consistent with participation rates for online surveys that are voluntary at the University of Waterloo. Out of a total of 311 students enrolled in the capstone project course, a total of 60 students completed the survey. This represented a participation rate of $19.3 \%$. This was very close to the $20 \%$ participation rate that was anticipated by the course coordinator.

\subsection{Response Quality}

The survey was designed to have some redundancy in its questions to allow a measure of response quality. In general, the overall impression assessments closely resembled the arithmetic mean of the assessments of the corresponding factors. No outliers or deviations of significance were noted.

The majority of the ratings were "Outstanding" or "Very Good". However, it is important to note that the entire range of ratings were used by the students in the survey. Ratings of "Outstanding", "Very Good", "Good", "Satisfactory", and "Unsatisfactory" were recorded for each question posed.

One interesting result to note was that students always rated their first team member higher than all other team members. This observation demonstrates that students have a "glass half-full" attitude. In other words, the first impression of students responding to the survey questions was the most positive one.

Another interesting result to note was that students tended to rate the overall impression of teamwork skills slightly higher than the overall impression of leadership skills. The reason for this observation is not clear. Students might have possess better teamwork skills. However, it might also be the case that students have a much higher expectation of leadership skills.

\subsection{Advantages of Anonymous Surveys}

One advantage of an anonymous survey of teamwork and leadership skills when compared to other assessment techniques is that it avoids forcing a change in the behaviour of the students. Since students have no direct interest in the results of the survey, they have no reason to manipulate the results. In other words, an honest assessment of team work and leadership skills can be obtained.

The use of an anonymous survey is also advantageous since it avoids the need to incorporate yet another deliverable into the marking scheme of the capstone project. This helps maintain the focus of the project courses on the project itself. It also reduces the amount of administration work required.

A final advantage of anonymous survey is that it does not require a substantial amount of effort to implement. A short survey, such as the one described in this paper, can be implemented in under an hour. 
Using a survey tool of a learning management system such as the one provided by Desire2Learn, results can be summarized and analyzed very quickly.

\subsection{Disadvantages of Anonymous Surveys}

One of the disadvantages of an anonymous survey is that the results might not always be representative of the entire class due to low participation rates in the survey. However, there is no reason to believe that the survey results are not representative of the entire class. Students do not have any direct interest in the results of the survey. There is no incentive to provide harsh or generous assessments. It might be possible to increase participation rates by providing students with an incentive for completing the survey. However, it is not clear if higher participation rates would improve the quality of the results or degrade them.

Another disadvantage of an anonymous survey is that the anonymous nature of the survey does not lend itself well to providing feedback to students who have done an exceptional job or those who have done a poor job. This disadvantage could be avoided by instituting a second assessment for the sole purpose of providing peer feedback to team members on their performance. Currently, such peer feedback is provided through the use of other mechanisms such as supervised meetings with the course coordinator. Providing feedback to individuals on the perceived quality of their teamwork and leadership skills was not a stated goal of this survey.

\section{CONCLUSIONS AND FUTURE WORK}

The anonymous survey of teamwork and leadership skills yielded the desired results for our program. Through the use of this assessment technique, the abilities of graduating students with respect to teamwork and leadership skills can be quantified in a meaningful way. Using these results, changes in teamwork and leadership skills can be tracked over time. A process of continuous program improvement will be implemented to use the compiled information to improve the quality of our program and our graduates.

In the future, the survey may be changed to add a self-assessment of teamwork and leadership skills. The results of this additional assessment could be used to establish a baseline for comparing peers. It might also be possible to perform adjustments to ratings to account for student assessors that tend to be harsh or generous with their ratings.
Finally, it would be interesting to investigate perceived teamwork and leadership skills early in a degree program. Using this information, it might be possible to quantify how much teamwork and leadership skills improve over the course of an undergraduate degree program. However, it might be difficult to discern a change in teamwork and leadership skills as student expectations are likely to increase substantially over the course of an undergraduate degree program.

\section{Acknowledgements}

The author wishes to acknowledge Dan Davison's extraordinary effort to design, develop, and implement the framework currently being used for outcomes assessment by the Department of Electrical and Computer Engineering at the University of Waterloo.

\section{References}

[1] CEAB. (2011) Accreditation Criteria and Procedures 2011. Canadian Engineering Accreditation Board. [Online]. Available: http://www.engineerscanada.ca/ files/w_Accreditation_Criteria_Procedures_2011.pdf

[2] Benjamin S Bloom, Taxonomy of Educational Objectives, Handbook I: The Cognitive Domain. New York: David McKay Co. Inc., 1956.

[3] (2013) Team Building App - Team Leadership App. Dale Carnegie Training. [Online]. Available: http://www.dalecarnegie.com/knowledge_center/tips/ leadership_series_part_2_of_3_team_leadership_app/

[4] Tanya Prive. (2012, December) Top 10 Qualities That Make A Great Leader. Forbes Magazine. [Online]. Available: http://www.forbes.com/sites/tanyaprive/2012/ 12/19/top-10-qualities-that-make-a-great-leader/

[5] (2012) Program Review and Accreditation - Background. Centre for Teaching Excellence, University of Waterloo. [Online]. Available: http://cte.uwaterloo.ca/teaching_ resources/OCAV/index.html

[6] W. Bishop, O. Nespoli, and W. Parker, "Rubrics for Accreditation and Outcomes Assessment in Engineering Capstone Projects," in Proceedings of the 2012 Canadian Engineering Education Association (CEEA12) Conference, June 2012. 